\title{
Caracterización térmica, mecánica y morfológica de fibras naturales colombianas con potencial como refuerzo de biocompuestos
}

\author{
William Javier Mora-Espinosa*, Bladimir Azdrúbal Ramón-Valencia \\ Grupo de Investigación en Ingeniería Mecánica, Departamento de Mecánica, Mecatrónica e Industrial, \\ Universidad de Pamplona, Pamplona, Colombia
}

\begin{abstract}
Resumen
Este estudio se enmarca en el campo de la ciencia e ingeniería de materiales enfocada al desarrollo de nuevos compuestos amigables con el medio ambiente. En este contexto, se evaluaron cinco fibras naturales colombianas: damagua (Poulsenia armata), guérregue, palma estera (Astrocaryum malybo), caña flecha (Gynerium sagitatum) e iraca (Carludovica palmata), con el fin de estudiar su viabilidad como refuerzo en materiales compuestos. Mediante diversas mediciones se determinaron las propiedades térmicas, mecánicas y morfológicas de las fibras naturales. El análisis térmico se hizo utilizando termogravimetría (TGA), y se demostró el carácter hidrofílico de las fibras y su estabilidad a elevadas temperaturas. Se ensayó la resistencia de las fibras a la tracción bajo condiciones de fuerzas axiales estáticas, y se encontraron variaciones en las propiedades mecánicas de cada uno de los especímenes. Las fibras de guérregue y caña flecha registraron valores de resistencia máxima competitivos muy similares a los reportados en otras investigaciones con fibras naturales, en tanto que las otras tres fibras tuvieron bajo desempeño. La microestructura de las fibras se examinó mediante microscopía electrónica de barrido (SEM), y las imágenes revelaron una morfología compuesta por el lumen y la pared celular con variación entre los tamaños, comportamiento éste asociado a las propiedades mecánicas de las fibras naturales estudiadas. En conclusión, dos fibras presentaron las mejores propiedades y cumplieron con las condiciones de estabilidad térmica y resistencia mecánica que las hace aptas como refuerzo en la fabricación de biocompuestos con matrices poliméricas. (C) 2017. Acad. Colomb. Cienc. Ex. Fis. Nat.
\end{abstract}

Palabras clave: Fibras naturales; Refuerzo; Propiedades mecánicas; Termogravimetría; Microscopía electrónica de barrido.

Thermal, mechanical, and morphological characterization of Colombian natural fibers as potential reinforcement for biocomposites

\begin{abstract}
This study was conducted in the field of materials science and engineering and focused on the development of new eco-friendly components. For this purpose, we evaluated five Colombian natural fibers: damagua (Poulsenia armata), quérregue (Astrocaryum standleyanum), palm mat (Astrocaryum malybo), wild cane (Gynerium sagitatum) and iraca (Carludovica palmata), to evaluate their viability as reinforcement in composite materials. We measured the thermal, mechanical and morphological properties of these natural fibers by using thermogravimetric analysis (TGA) for the thermal analysis, which showed their hydrophilic character and their stability at high temperatures. We also tested their resistance to traction under static axial forces and we found variations in their mechanical properties. We found that guérregue and wild cane fibers showed maximum competitive strength values, similar to those found in other studies with natural fibers, while the other three fibers had a poor performance. Scanning electron microscopy (SEM) was used to examine the microstructure of the fibers showing a morphology composed of lumen and cell wall with variations among sizes, a behavior which is linked to their mechanical properties. As a result, we found that two of the fibers complied with the conditions of thermal stability and tensile stress to be used as reinforcement in the manufacturing of biocomposites with polymeric matrices. (C) 2017. Acad. Colomb. Cienc. Ex. Fis. Nat.
\end{abstract}

Key words: Natural fibers; Reinforcement; Mechanical properties; Thermogravimetric analysis; Scanning electron microscopy.

\section{Introducción}

Actualmente el uso de fibras naturales como refuerzo de materiales compuestos ha despertado un gran interés en la comunidad científica, dadas sus propiedades específicas frente a otras fibras de origen sintético (Fiore, et al., 2011). Todas las fibras lignocelulósicas presentan microestructuras cristalinas formadas por celulosa y microestructuras en espiral conectadas por lignina amorfa y hemicelulosa (Monteiro, et al., 2012). Mediante microscopia electrónica

\footnotetext{
*Correspondencia:

William Javier Mora-Espinosa, wjme11@gmail.com

Recibido: 22 de septiembre de 2017
}

Aceptado: 14 de diciembre de 2017 
de barrido (SEM) se ha determinado que cuanto menor sea el ángulo de las fibrillas de celulosa, la respuesta mecánica de la fibra aumenta (Saravanakumara, et al., 2013).

Algunas fibras naturales han entrado al mercado como refuerzo en materiales compuestos para la fabricación de partes, principalmente en el sector automotriz y de empaques (Kommula, et al., 2014). Los compuestos reforzados con fibras naturales presentan una alternativa más ecológica, y por ello han desplazado los materiales convencionales en algunas aplicaciones industriales, en especial los compuestos elaborados con fibras sintéticas de vidrio (Chandramohan \& Marimuthu, 2011), pues constituyen alternativas como lo demuestran algunas investigaciones adelantadas con fibras naturales como Prosopis juliflora (Saravanakumara, et al., 2013), lino-plátano (Srinivasan, et al., 2014), curauá (Spinacé, et al., 2009), Cissus (Indran, et al., 2014), Spartium junceum (Kovacevic, et al., 2015), Arundo donax (Fiore, et al., 2014), cáñamo de Marruecos (Elkhaoulani, et al., 2013) y palma de azúcar (Arenga pinnata) (Ishaka, et al., 2013), entre otras, lo cual ha facilitado una amplia gama de fibras útiles como refuerzo.

Colombia tiene el $0,7 \%$ de la superficie continental mundial y el $10 \%$ de la biodiversidad del mundo, lo que representa entre 45.000 y 55.000 especies de plantas, cifras muy similares a las de Brasil, que cuenta con 55.000 (Romero, et al., 2009); este potencial solo es aprovechado en la confección de artesanías por una población constituida en su mayoría de familias campesinas y grupos indígenas (Linares, et al., 2008). Entre los principales usos de las fibras naturales colombianas está la confección de bolsos, sombreros, tapetes, canastos y costales que se utilizan como empaques de alimentos (Artesanías de Colombia, 2011).

En diversos estudios se han reportado fibras colombianas usadas como refuerzo. La fibra de plátano fue tratada en una matriz de resina de poliéster y se registró un aumento de la resistencia mecánica frente a la resina pura y una disminución de la absorción de agua (Rodríguez, et al., 2014). Las fibras de cumare se utilizaron como refuerzo en una matriz de ácido láctico, y se registró poca adhesión entre la fibra y la resina, con efectos negativos en las propiedades mecánicas (Maranon \& Páez, 2011). En otro estudio, se embebieron fibras de fique en una matriz de poliéster insaturado, y se evidenció un desprendimiento de las capas exteriores de los laminados después de ser ensayados bajo tracción (Contreras, et al., 2009). Asimismo, se empleó la fibra de guadua como refuerzo de matrices poliméricas y con ello se incrementó la resistencia mecánica en los ensayos de compresión e impacto de muestras con $10 \%$ de fibra modificada (Cuellar \& Muñoz, 2010).

La propuesta de investigación del presente estudio consistía en el análisis de cinco fibras naturales autóctonas de Colombia para evaluar su posible uso como refuerzo en biocompuestos. Para tal fin, se caracterizó la estabilidad térmica de las fibras con un analizador termogravimétrico y se obtuvieron las curvas pérdida de peso (TG) y la primera derivada de la pérdida de peso (DTG). El comportamiento mecánico de las fibras se evaluó mediante ensayos de tensión uniaxial ajustados a la normas de la American Society for Testing and Materials (ASTM). La morfología se observó con un microscopio electrónico de barrido (SEM), con el cual se obtuvieron imágenes de las microestructuras de cada una de las fibras naturales. A partir de los resultados obtenidos con cada una de las técnicas, se demostró que estas podrían ser una alternativa para el desarrollo de nuevos materiales de bajo costo y amigables con el medio ambiente.

\section{Materiales y métodos}

Las cinco fibras estudiadas fueron: Poulsenia armata (Miq.) Standley (1933), conocida como damagua, proveniente de un árbol que alcanza hasta $40 \mathrm{~m}$ de altura y más de $100 \mathrm{~cm}$ de diámetro (Castillo, 2010), la fibra se usa para billeteras, bolsos, flores y sombreros (Cardona, et al., 2005); Astrocaryum standleyanum (Borgtoft, 1994) o palma guérregue, con un tallo espinoso de hasta 12 $\mathrm{m}$ de altura y 15 a $20 \mathrm{~cm}$ de diámetro, cuya fibra se usa para elaborar canastos, cántaros, platos, bandejas, bolsos, sombreros, pulseras, aretes y collares (Galeano, et al., 2010); Astrocaryum malybo (Linares, et al., 2008) o palma estera, cuyas hojas proporcionan la materia prima para la industria de las esteras o petates (Linares, et al., 2008), (Calderón, et al., 2005), así como individuales, portavasos y piedecamas (Bernal \& Galeano, 2013); Carludovica palmata (Escobar, 2001) o palma iraca, planta que alcanza hasta los $5 \mathrm{~m}$ de alto y tiene hojas en forma de abanico de 50 por $80 \mathrm{~cm}$ (Artesanías de Colombia, 2011), de las cuales se extrae la fibra que, una vez procesada, sirve para tejer sombreros (Escobar, 2001), carrieles y alpargatas, pavas, individuales, sombrillas, bandejas, cinturones y bolsos (Artesanías de Colombia, 2011), y Gynerium sagittatum (Pohl, 1983) o caña flecha, con tallos de 5 o $6 \mathrm{~m}$ de altura y 2 o $3 \mathrm{~cm}$ de diámetro (Linares, et al., 2008), de cuyas hojas se aprovecha la nervadura central para obtener la fibra usada para la trenza del sombrero, así como el bagazo, que se usa como forraje (Artesanías de Colombia, 2011). Una descripción más detallada de las plantas y fibras procesadas se muestra en la Tabla 1S, https://www.raccefyn.co/index. php/raccefyn/article/downloadSuppFile/525/2411.

El proceso de extracción varía según la naturaleza de las fibras. En el caso de la palma quérregue, la palma estera y la palma de iraca, se cosechan las hojas jóvenes o cogollos, se separan manualmente en tiras más finas que se secan al sol (Artesanías de Colombia, 2011), (Ventas artesanías en Werregue, 2012), (Artesanías de Colombia, et al., 2008). El trabajo con la damagua se inicia con el corte del árbol, luego se abre la corteza, se extrae la fibra en forma de tela, se lava y se seca al sol (Ramírez, et al., 2011). En cuanto a la cañaflecha, se seleccionan las hojas maduras y se recolectan, se separan en filamentos más delgados y se llevan a un proceso de cocción y secado (Linares, et al., 2008). La extracción y la preparación de las fibras generalmente está a 
cargo de las mujeres de los diversos grupos étnicos y de artesanos de tradición ancestral, actividad que se desarrolla en todo el territorio del país (Quiñones, 2000). En la Figura 1S, https://www.raccefyn.co/index.php/raccefyn/article/download SuppFile/525/2403, se muestra el proceso de extracción de la fibra de palma guérregue.

Termogravimetría. La estabilidad térmica de las fibras naturales se evaluó mediante la técnica de termogravimetría (TGA) en un equipo modelo SDT Q600 de TA Instruments. Las muestras se trituraron y compactaron entre 5 y $10 \mathrm{mg}$ y posteriormente se programaron rampas de calentamiento a partir de temperatura ambiente hasta alcanzar los $600{ }^{\circ} \mathrm{C}$ a una velocidad de calentamiento de $10{ }^{\circ} \mathrm{C} / \mathrm{min}$. Durante el barrido las muestras se controlaron en una atmósfera inerte de nitrógeno.

Ensayos de tracción. Las propiedades mecánicas se llevaron a cabo en la máquina universal Shimatzu con capacidad de $600 \mathrm{kNI}$, utilizando un juego de accesorios para pruebas de tensión en fibras. Los parámetros se ajustaron a la norma de la ASTM D3822/D3822M - 14 (Standard Test Method for Tensile Properties of Single Textile Fibers), en condiciones de temperatura ambiente, humedad relativa de $60 \%$ y una velocidad de deformación de $1 \mathrm{~mm} / \mathrm{min}$ para 20 especímenes por fibra.

Microscopía electrónica de barrido. La morfología estructural de las fibras estudiadas se analizó usando un microscopio electrónico de barrido de marca JEOL, modelo JSM 6490-LV, con una tensión de $30 \mathrm{kV}$ y aislamiento por alto vacío. Previamente, las muestras se habían cubierto con una capa delgada de oro para mejorar la conductividad.

Análisis estadístico. El diseño de los experimentos para determinar el número adecuado de réplicas en los ensayos de tracción de las fibras se hizo mediante la ecuación 1 (Lozano, 2011), con un valor de confianza superior a $99 \%$. Las fibras se encontraban totalmente secas y se cortaron con la misma longitud.

$$
n=\frac{\mathrm{W}-W^{2} * Z_{\beta}+1,4 * Z_{\alpha}^{2}}{W^{2}}
$$

donde $\mathrm{n}=$ número mínimo de muestras, observaciones o réplicas que deben efectuarse en el estudio; $Z \alpha=$ valor correspondiente al nivel de confianza asignado (riesgo de cometer un error de tipo I); $Z \beta$ = valor correspondiente al poder estadístico o potencia asignada a la prueba (riesgo de cometer un error de tipo II); $\mathrm{W}$ = rendimiento mínimo esperado, eficiencia mínima esperada o diferencia mínima observable. Los valores $Z \alpha$ y $Z \beta$ para diferentes niveles de confianza se obtuvieron de la Tabla 1.

Los valores de la tabla para un nivel de confianza de $99 \%$ y un poder estadístico de $90 \%$ se utilizaron en la ecuación y se obtuvo un número de 18 réplicas, cercano a los 20 especímenes que exige la norma ASTM D3822/D3822M 14, con lo cual se validó esta cantidad de réplicas por fibra para determinar las propiedades mecánicas.
Se aplicaron análisis de estadística descriptiva a los resultados de los ensayos con las cinco fibras para determinar los promedios y la desviación estándar de las diversas propiedades.

\section{Resultados}

Análisis térmico. El comportamiento térmico de las fibras naturales ha evidenciado poca estabilidad, lo cual genera su degradación durante algunos procesos de fabricación de materiales compuestos (Mohanty, et al., 2000). Su carácter hidrófilo no permite la formación de uniones interfaciales fuertes con la matriz, lo cual resulta en una reducción de las propiedades mecánicas (Girisha \& Srinivas, 2012). La técnica de TGA permite obtener información sobre la cantidad de humedad que puede retener una fibra natural, anticipando la compatibilidad con la matriz polimérica (Miranda, et al., 2013).

El comportamiento térmico de las fibras de damagua, guérregue, palma estera, caña flecha e iraca, se muestra a continuación mediante las curvas de pérdida de peso (TG) y la primera derivada de la pérdida de peso (DTG), ambas en función de la temperatura.

En la curva TG de la damagua (Figura 1a), se observa una primera pérdida de la masa atribuida a la evaporación de la humedad (Miranda, et al., 2013) en un rango de temperaturas de 25 a $125{ }^{\circ} \mathrm{C}$, con una pérdida de peso de $11,65 \%$. Posteriormente, en la curva DTG se muestran cuatro etapas de descomposición térmica: un pico I de menor intensidad, no muy característico, alrededor de los $167^{\circ} \mathrm{C}$, reportado en algunos artículos como el inicio de la pirolisis de la lignina (Indran, et al., 2014); un pico II a $257^{\circ} \mathrm{C}$, con una pérdida de la masa de 10,86\%, asociada con la despolimerización de la hemicelulosa, las pectinas y el glucósido, cuya estructura es muy semejante a la celulosa (Moriana, et al., 2014); el pico III, relacionado con la descomposición de la $\alpha$-celulosa (Fiore, et al., 2014), se presenta a $344{ }^{\circ} \mathrm{C}$ con una pérdida de $47,95 \%$ de la masa, y, por último, se observa el pico IV a los

Tabla 1. Valores $\alpha, Z \alpha, \beta$ y Z $\beta$ para diversos niveles de confianza y poder estadístico

\begin{tabular}{|c|c|c|}
\hline Valores & \multicolumn{2}{|c|}{ Nivel de confianza (1- $\alpha)$} \\
\hline$\alpha$ & $(\%)$ & $\mathbf{z \alpha}$ \\
\hline 0,050 & 95,0 & 1,960 \\
\hline 0,025 & 97,5 & 2,240 \\
\hline 0,010 & 99,0 & 2,576 \\
\hline Valores & \multicolumn{2}{|c|}{ Poder estadístico $(1-\beta)$} \\
\hline$\beta$ & $(\%)$ & $\mathbf{Z \beta}$ \\
\hline 0,20 & 80,0 & 0,842 \\
\hline 0,15 & 85,0 & 1,036 \\
\hline 0,10 & 90,0 & 1,282 \\
\hline
\end{tabular}



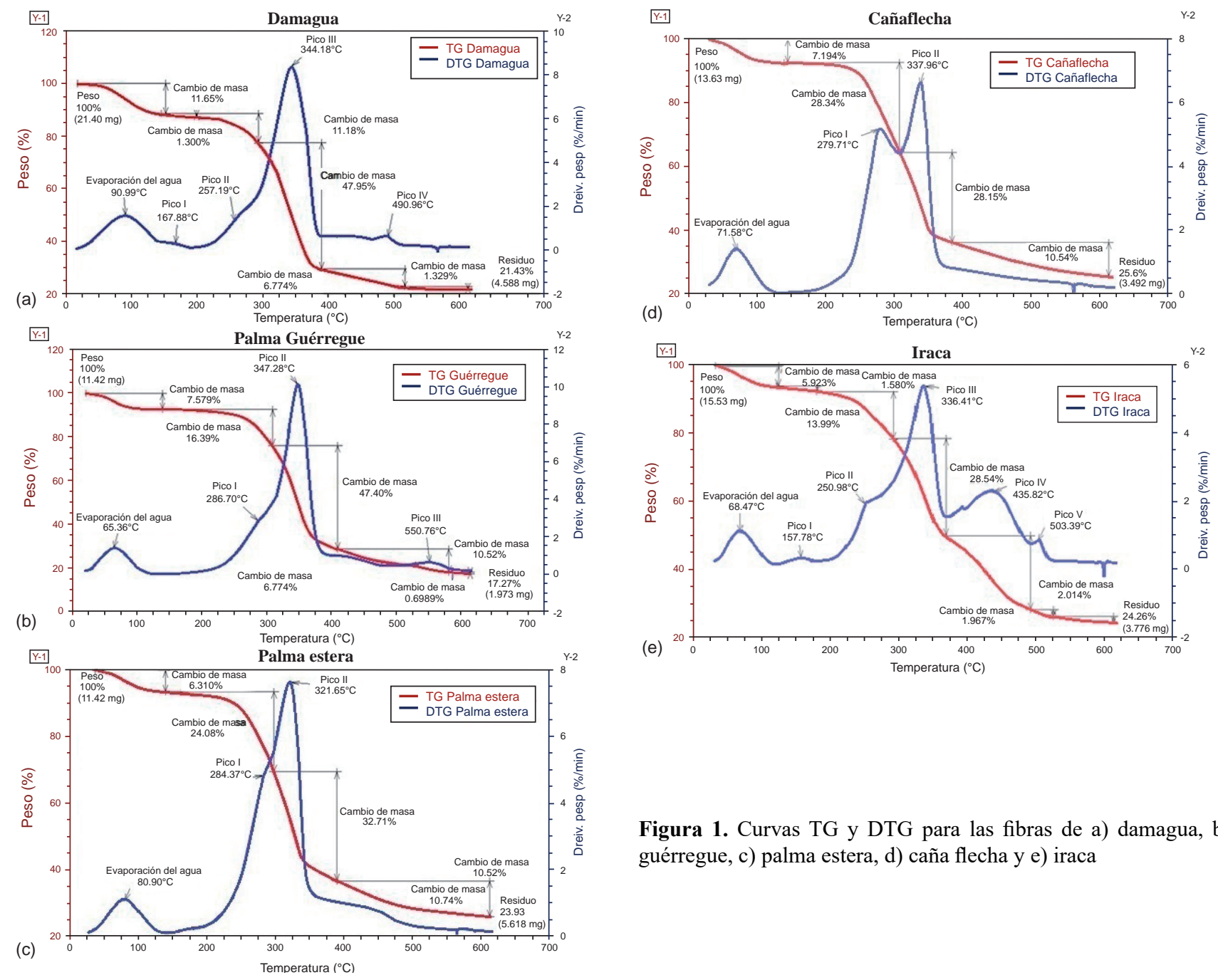

Figura 1. Curvas TG y DTG para las fibras de a) damagua, b) guérregue, c) palma estera, d) caña flecha y e) iraca

$491{ }^{\circ} \mathrm{C}$, con una pérdida de masa de $6,8 \%$, que corresponde a la degradación final de la lignina y la oxidación de residuos carbonizados de la fibra (Das, et al., 2000).

En la fibra de guérregue (Figura 1b), la curva TG muestra una primera pérdida de la masa de 7,58 \%, entre los 25 y los $125^{\circ} \mathrm{C}$, asociada con la evaporización del agua. En la curva DTG se observan solo tres picos: el pico I se atribuye a la despolimerización de la hemicelulosa, las pectinas y el glucósido (Moriana, et al., 2014), a una temperatura alrededor de los $287^{\circ} \mathrm{C}$, con una pérdida de masa de $16,39 \%$; el pico II está relacionado con la descomposición de la $\alpha$-celulosa en torno a los $347^{\circ} \mathrm{C}$ y a un consumo de la masa total de $47,4 \%$, resultados similares a los reportados en el estudio térmico de la fibra natural de la okra (DeRosa, et al., 2011); y, por último, se observa el pico III a los $551^{\circ} \mathrm{C}$, con $10,52 \%$ de pérdida de masa, que representa la degradación final de la lignina, ya que debido a la compleja estructura de esta fase, esta se degrada en todo el rango de temperatura (Liu, et al., 2004).

En cuanto a la palma estera (Figura 1c), la curva TG presentó un primer cambio en la masa de 6,31 \%, por la humedad que se evaporó en la fibra. Los siguientes cambios se produjeron en tres estadios: en el pico I, se degradaron la hemicelulosa, las pectinas y el glucósido en torno a los $284{ }^{\circ} \mathrm{C}$, con una pérdida de masa de $24,08 \%$; en el pico II, se descompuso la $\alpha$-celulosa a los $321^{\circ} \mathrm{C}$, con una pérdida de masa de $32,71 \%$, y el pico III correspondió a la fragmentación de la lignina, con 10,74 \% de pérdida de masa.

Al igual que en las otras, en la fibra de caña flecha (Figura 1d) la curva TG reveló un primer descenso de la masa relacionado con la evaporación de la humedad en torno a una temperatura entre los 25 y los $125^{\circ} \mathrm{C}$, con una pérdida de peso de 7,04\%. En la curva DTG también se evidenciaron tres etapas de descomposición. En el pico I, a los $279{ }^{\circ} \mathrm{C}$ hubo una pérdida de masa de $27,37 \%$ asociada con la relajación de las estructuras de hemicelulosa, pectinas y glucósido; el pico II correspondió a la desintegración de la fase cristalina de la $\alpha$-celulosa a una temperatura de $338^{\circ} \mathrm{C}$, con una de pérdida de masa de $28,88 \%$, y, por último, entre los 375 y los $600{ }^{\circ} \mathrm{C}$, la pérdida de masa fue de $10,86 \%$, rango correspondiente a la degradación de la lignina y la oxidación de residuos carbonizados (García-Pérez, et al., 2001; D'Almeida, et al., 2006; Yang, et al., 2007; Spinance, et al., 2009). 
En cuanto a la iraca (Figura 1e), la evaporización de la humedad se apreció en la curva TG en un rango de 25 a $110^{\circ} \mathrm{C}$, con una pérdida de masa de 5,92\%. Después se presentó un comportamiento poco frecuente en las fibras naturales, con la presencia de cinco picos en la curva DTG durante el proceso de descomposición térmica, resultados similares a los reportados en el análisis térmico mediante TGA de la fibra del tallo de Cissus quadrangularis (Indran \& Raj, 2015). El pico I se observó a los $158^{\circ} \mathrm{C}$, con una pérdida de masa de 1,58 \%, relacionada con el inicio de la pirolisis de la lignina. A continuación, se observó el pico II, correspondiente a la degradación de la hemicelulosa y los enlaces glucosídicos, a $251{ }^{\circ} \mathrm{C}$ y con una pérdida de masa de $13,99 \%$. El pico III se produjo a los $336^{\circ} \mathrm{C}$, con una pérdida de masa de $28,54 \%$, lo que se atribuye a la descomposición de la $\alpha$-celulosa (Azwa, et al., 2013). Posteriormente, se evidenciaron los picos IV y V a los $436{ }^{\circ} \mathrm{C}$ y $503{ }^{\circ} \mathrm{C}$, y con 21,74 y $2,01 \%$ de pérdida de masa, respectivamente, lo cual se asoció a la degradación final de la lignina y la oxidación de los residuos carbonizados de la fibra (Saravanakumara, et al., 2013).

Debido al carácter hidrofílico de las fibras, en todas se observó un porcentaje significativo de humedad retenida, sobre todo en la damagua, con una mayor adsorción de agua $(11,65 \%)$, en tanto que la iraca registró la menor $(5,92 \%)$ en un rango de 25 a $125{ }^{\circ} \mathrm{C}$, comportamiento parecido al de las fibras de Althaea $\left(25-125^{\circ} \mathrm{C}\right)$ (Sarikanat, et al., 2014), Areca $\left(30-100{ }^{\circ} \mathrm{C}\right)$ (Yusriah, et al., 2014), Proporis $\left(25-110^{\circ} \mathrm{C}\right)$ (Saravanakumara, et al., 2013), caracua (25$\left.150{ }^{\circ} \mathrm{C}\right)$ (Dai, et al., 2010), okra $\left(30-110{ }^{\circ} \mathrm{C}\right)$ (DeRosa, et al., 2011) y Cortaderia selloana $\left(50-150{ }^{\circ} \mathrm{C}\right)$ (JordáVilaplana, et al., 2017). Este fenómeno sugiere que las fibras deben pasar por un tratamiento químico o térmico para optimizar la adherencia al usarlas como refuerzo con matrices poliméricas.

La estabilidad térmica alcanzó a superar los $200{ }^{\circ} \mathrm{C}$ en las cinco fibras, es decir, iniciaron su proceso de descomposición por encima de este valor. En la fibra guérregue se inició a los $219,14{ }^{\circ} \mathrm{C}$, valor cercano al obtenido en otras fibras como la de la alcachofa $\left(230^{\circ} \mathrm{C}\right)$, el bambú $\left(214{ }^{\circ} \mathrm{C}\right)$, el bagazo $\left(222^{\circ} \mathrm{C}\right)$ (Fiore, et al., 2011), la okra $\left(220^{\circ} \mathrm{C}\right)$, el cáñamo $\left(250^{\circ} \mathrm{C}\right)$, la curauá $\left(230^{\circ} \mathrm{C}\right)$, el kenaf $\left(219^{\circ} \mathrm{C}\right.$ ), el yute $\left(205^{\circ} \mathrm{C}\right)$ (DeRosa, et al., 2011), Juncus effusus L. $\left(220^{\circ} \mathrm{C}\right)$ (Maache, et al., 2017) y el cactus de aloe vera del Sahara $\left(238^{\circ} \mathrm{C}\right)$ (Balaji \& Nagarajan, 2017). Este factor determina las condiciones de temperatura para el proceso de transformación en materiales compuestos con matrices poliméricas, de manera que solo se podrán utilizar termoplásticos que no superen la temperatura de degradación de las fibras.

Propiedades mecánicas. Las pruebas mecánicas se ajustaron a la norma ASTM D3822M - 14, la cual define algunas condiciones para los ensayos, como la velocidad de deformación de $1 \mathrm{~mm} / \mathrm{min}$ y un número de 20 especímenes de ensayo por fibra, cantidad validada mediante la ecuación 1,descrita en el análisis estadístico (Lozano, 2011).
En la Figura 2 se presentan las curvas de tendencia del comportamiento promedio para el esfuerzo y la deformación de cada fibra.

En los diagramas se observa un comportamiento dúctil en las fibras de guérregue, caña flecha y palma estera, representado por una zona elástica (lineal) y una zona plástica (no lineal). La zona elástica se asoció con la deformación de la pared celular (microfribrillas de celulosa) y la plástica con la delaminación de la celulosa y la orientación de la fase amorfa (lignina, pectina y hemicelulosa) (Fiore, et al., 2014). Las fibras de iraca y damagua solo evidenciaron un estado elástico-lineal.

En la Tabla 2 se presentan los valores promedio del módulo elástico, la resistencia máxima y el porcentaje de deformación de las fibras estudiadas. Los valores se obtuvieron de los promedios registrados en los 20 ensayos, con su respectiva desviación estándar.

Con base en estos datos (Tabla 2), las fibras se clasificaron en orden descendente con respecto a los valores obtenidos en la resistencia máxima a la tracción, dada la importancia que representa este parámetro en el diseño, la selección y la aplicación de piezas obtenidas a partir de materiales compuestos. Los valores de la desviación estándar obtenidos para el esfuerzo fueron relativamente bajos comparados con los de otras fibras como el lino $(1000 \pm 500 \mathrm{MPa})$ (Hughes, 2012), okra (233,8 $\pm 126,4$ MPa) (DeRosa, et al., 2011) y ramio $(586,5 \pm 351,5 \mathrm{MPa})$ (Sarikanat, et al., 2014), lo que permite validar los datos obtenidos a partir de las 20 réplicas ajustadas a la norma.

La evaluación de las propiedades mecánicas de las fibras naturales se hizo teniendo en cuenta los parámetros obtenidos en el diagrama de esfuerzo y deformación por separado.

El comportamiento del módulo de Young o módulo de elasticidad (Figura 3a) está asociado al ordenamiento de las microfibrillas de la pared celular en la misma dirección de la aplicación de la fuerza. Los valores obtenidos variaron entre fibras, con altos módulos de rigidez en el guérregue y la iraca, con 5,85GPa y 7,61 GPa, respectivamente. Resultados similares se han reportado con las de fibras del algodón ( 5

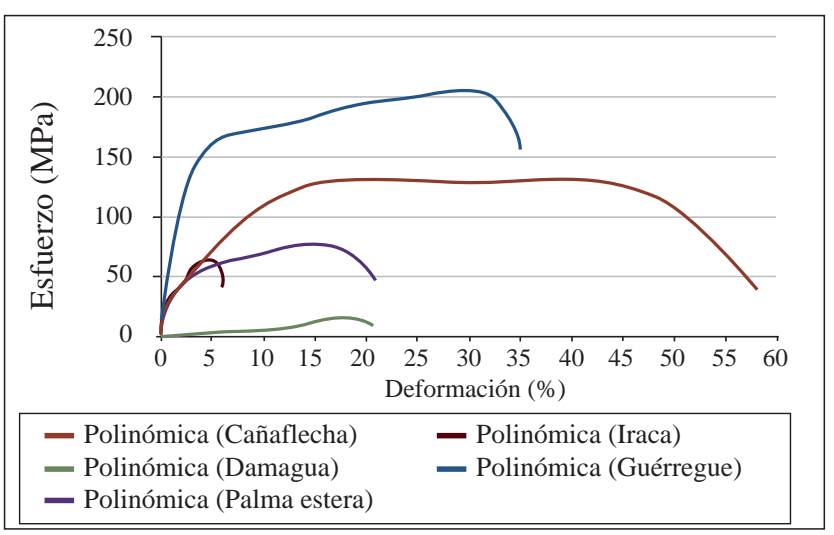

Figura 2. Diagrama de esfuerzo y deformación de cinco fibras colombianas 
Tabla 2. Propiedades mecánicas de las fibras de guérregue, caña flecha, palma estera, iraca y damagua

\begin{tabular}{lccccc}
\hline $\begin{array}{c}\text { Área transversal } \\
\left(\mathbf{m m}^{\mathbf{2}}\right)\end{array}$ & $\begin{array}{c}\text { Tipo } \\
\text { de fibra }\end{array}$ & $\begin{array}{c}\text { Longitud } \\
\text { calibrada } \\
\mathbf{( m m )}\end{array}$ & $\begin{array}{c}\text { Módulo } \\
\text { elástico } \\
\mathbf{( G P a )}\end{array}$ & $\begin{array}{c}\text { Esfuerzo máximo } \\
\text { a tensión } \\
(\mathbf{M P a})\end{array}$ & $\begin{array}{c}\text { Deformación } \\
\mathbf{( \% )}\end{array}$ \\
\hline $0,27 \times 1,40$ & Guérregue & 20 & $5,85 \pm 2,50$ & $249,11 \pm 97,40$ & $36,29 \pm 26,70$ \\
\hline $0,16 \times 2,00$ & Caña flecha & 20 & $3,53 \pm 1,60$ & $168,23 \pm 59,80$ & $43,17 \pm 17,70$ \\
$0,27 \times 2,00$ & Palma estera & 20 & $2,53 \pm 1,20$ & $104,73 \pm 44,90$ & $31,07 \pm 17,80$ \\
\hline $0,4 \times 0,74$ & Iraca & 20 & $7,61 \pm 3,10$ & $100,89 \pm 39,30$ & $10,33 \pm 5,30$ \\
$0,93 \times 2,00$ & Damagua & 20 & $0,37 \pm 0,30$ & $18,78 \pm 7,30$ & $23,78 \pm 10,30$ \\
\hline
\end{tabular}

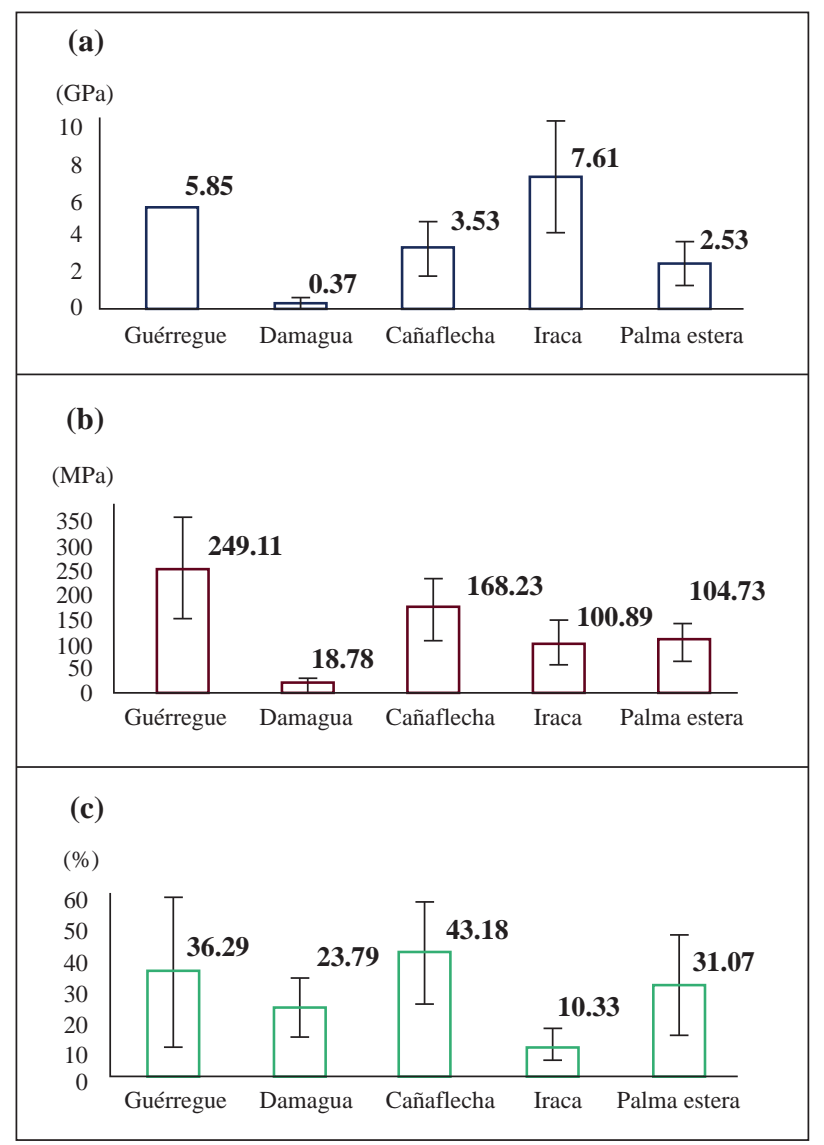

Figura 3. Propiedades mecánicas de las fibras naturales. a) Módulo de Young. b) Esfuerzo máximo. c) Porcentaje de deformación

-12 GPa), el coco (4 - $6 \mathrm{GPa}$ ) y Sansevieria cylindrica (6 - 8 GPa) (Indran \& Raj, 2015). Las fibras de palma estera y caña flecha presentaron módulos de 2,53 GPa y 3,53 GPa, respectivamente. Por último, la fibra de damagua presentó el módulo más bajo debido a su estructura de fibras cortas y discontinuas con uniones débiles entre sí.

La resistencia máxima se calculó a partir del esfuerzo último que soporta la fibra cuando es sometida a una tensión axial en tracción. Esta conducta puede variar entre las fibras debido a su comportamiento mecánico, es decir, en aquellas que solo presentan una zona elástica lineal, la resistencia máxima es igual al esfuerzo de rotura, como sucede con las fibras de iraca y damagua. Por otro lado, en las que presentan un comportamiento elástico-plástico, la resistencia máxima se determina cuando la fibra ha experimentado cierto grado de deformación, como sucedió con las fibras de guérregue, caña flecha y palma estera.

Se evidenciaron valores elevados de la resistencia máxima de las fibras (Figura 3b) de guérregue $(249,11 \mathrm{MPa})$, seguida de la caña flecha $(168,23 \mathrm{MPa})$. Estos valores son similares a los obtenidos en la fibra de ramio (220-938 MPa), de palma de aceite (248 MPa) y Palmyrah (180 -215MPa) (Indran, et al., 2015). Las fibras de iraca y palma estera mostraron esfuerzos de 100,89 MPa y 168,23 MPa, respectivamente. Por último, la fibra de damagua fue la de menor resistencia a la tensión por las razones ya anotadas en los cometarios sobre el módulo de elasticidad.

El comportamiento de la deformación está influenciado principalmente por la orientación de las fases cristalina de la celulosa y las fases amorfas de la lignina y la hemicelulosa en la dirección de la aplicación de la tensión axial, dando como resultado un material de carácter anisotrópico. (Fiore, et al., 2014).

Se encontraron diferencias en la deformación de las fibras analizadas (Figura 3c). La más elevada se registró en la fibra de la caña flecha, con $43,17 \%$, resultados similares a los reportados con las fibras de la hierba de mar (13 - 26,6\%) y la piasava (7,8-21,9\%) (Indran, et al., 2014). Las fibras de guérregue y palma estera registraron porcentajes de 36,29 y $31,07 \%$ en la deformación, respectivamente. Las fibras de damagua e iraca, con un comportamiento frágil, obtuvieron los menores porcentajes en la elongación, con 23,78 y $10,33 \%$, respectivamente.

Por último, de las cinco fibras analizadas, la de guérregue (249 MPa) presentó una resistencia a la tracción alta, lo cual evidenció un comportamiento cercano al de otras fibras naturales utilizadas como refuerzo en la elaboración de biocompuestos sometidos a cargas moderadas, entre ellas la de coco (220 MPa), la de cascarilla de arroz (225 MPa), de palma de aceite (248 MPa) y de caña de azúcar (290 MPa). También la de caña flecha (168,23 MPa) se aproximó a la de Palmyrah (180 MPa) y superó a las de coco verde (134,3 $\mathrm{MPa}$ ), de ceiba pentandra (45 MPa) y de palma datilera (97 $\mathrm{MPa})$, como se aprecia en la Figura 4. 


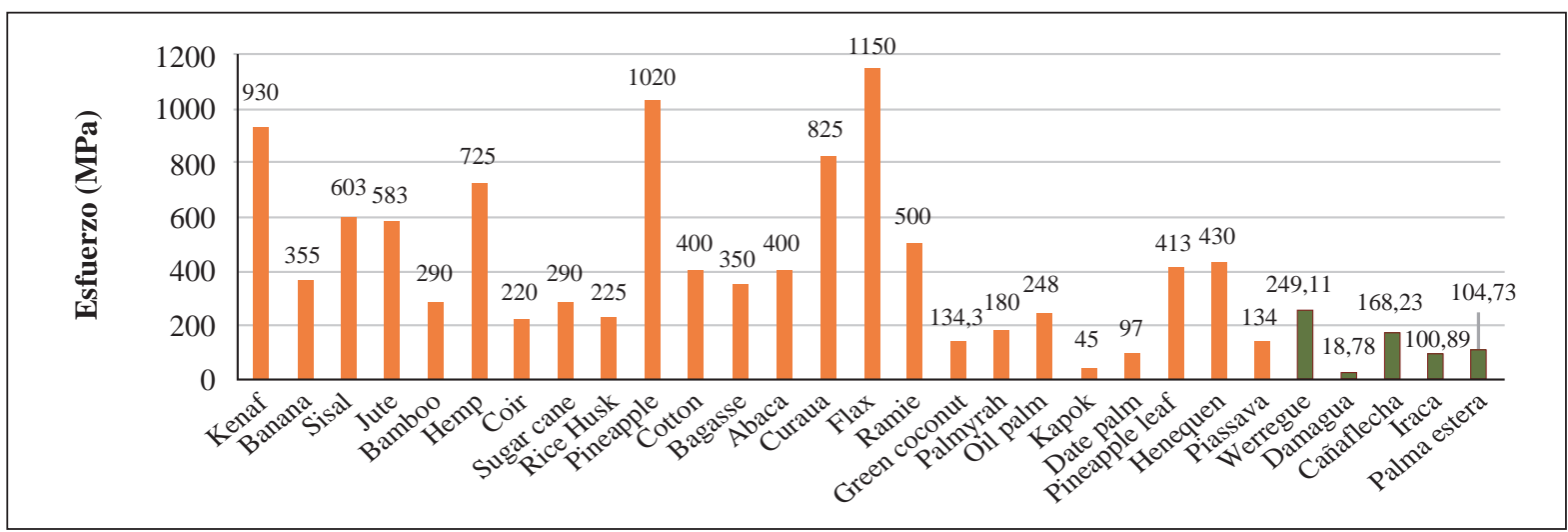

Figura 4. Esfuerzo máximo promedio de tracción en diversas fibras naturales (Arpitha \& Yogesha, 2017; Müssig-Jörg, 2010) comparado con el de fibras colombianas

Estos resultados permiten proponer estas dos fibras como posibles candidatas para refuerzos naturales en la fabricación de materiales compuestos, tanto en matrices termoestables como termoplásticas. Las otras tres fibras poseen valores muy bajos de resistencia, lo cual limita sus posibles aplicaciones como refuerzo.

Análisis morfológico. El análisis estructural de las fibras naturales se realizó mediante microscopía electrónica de barrido (SEM), con el fin de diferenciar la pared celular, el lumen y la laminilla media que conforman la estructura interna de la fibra. Las cadenas de celulosa están integradas por entre 30 y 100 moléculas con un ángulo microfibrilar de $0^{\circ}$ a $2^{\circ}$ de inclinación y son las encargadas de proporcionarle resistencia mecánica a la fibra (Dai \& Fan, 2010; Rong, et al., 2001). Las paredes celulares primarias, secundarias y terciarias se definen por la concentración de los materiales constituyentes y el ángulo microfibrilar, y se unen por la laminilla media (LM) constituida por lignina y hemicelulosa (Dai, et al., 2010). Las diferencias entre fibras se dan en el número de células, el tamaño de las paredes celulares y el lumen o agujero responsable del transporte de humedad y nutrientes (Thygesen, 2006), así como del área de sección transversal, con diversas características y comportamientos mecánicos (Alves, et al., 2013).

El comportamiento mecánico está ligado al rango elástico (casi lineal), a la resistencia de las paredes celulares y la laminilla media, zona donde soportan la carga sin delaminarse; en el rango plástico ocurre la delaminación tanto de las paredes primaria y secundaria como de la unión entre ellas (LM) (Guo, et al., 2014). Esta unión entre células presenta un comportamiento elástico-plástico en todas las fibras naturales, el cual está relacionado con la resistencia mecánica de la fase amorfa compuesta por la lignina y la pectina, así como de la fase cristalina asociada con la celulosa (Fuentes, et al., 2017).

En la morfología de la sección transversal de la fibra de damagua (Figura 5a), se observaron agujeros de gran tamaño, de entre 10 y $25 \mu \mathrm{m}$, que corresponden al lumen, lo cual permitió confirmar los resultados obtenidos en el TGA con respecto a su gran capacidad para retener agua. Por otro lado, la microestructura reveló espesores de 3 y $5 \mu \mathrm{m}$, lo cuales correspondían a la pared celular, lo cual permitió corroborar los bajos valores obtenidos en las propiedades mecánicas debido a la relación entre el espesor de la pared celular y el tamaño del lumen. Con respecto a la laminilla media, esta no se distinguió fácilmente entre las paredes celulares.

Para la morfología de la sección transversal de la fibra de palma guérregue (Figura 5b), con X 1,100 aumentos, en la SEM no se evidenciaron lúmenes, los cuales solo se lograron definir claramente con acercamientos incluso de X3000. La microestructura mostró una buena relación entre la pared celular y el lumen, lo que permitió obtener una mayor área para resistir las fuerzas axiales en tensión, debido a que sus componentes internos y microfibrillas distribuyeron mejor el esfuerzo.

La microestructura de la fibra de palma estera (Figura 5c) se analizó con varios aumentos. En las imágenes se distinguió fácilmente el lumen, con un tamaño aproximado de 4 a $6 \mu \mathrm{m}$, mientras que la pared celular osciló entre 1 y $3 \mu \mathrm{m}$, y la laminilla media entre células no fue muy visible. La fibra de palma estera presentó una resistencia similar a las fibras provenientes del tallo, lo cual se debe principalmente a que las microfibrillas de celulosa de las paredes celulares son más abundantes y el ángulo que poseen es bajo, y ello permitió que se alinearan en la dirección del esfuerzo (Pickering, et al., 2016).

En cuanto a la fibra de caña flecha (Figura 5d), las imágenes revelaron microconstituyentes que forman la estructura interna. Se observó una pared celular gruesa con espesores que oscilaban entre 4 y $6 \mu \mathrm{m}$, con variaciones en los diámetros del lumen de entre 1 y $3 \mu \mathrm{m}$, características que hacen que la fibra posea mayor área para soportar el esfuerzo al concentrar una mayor cantidad de microfibrillas de celulosa. Las imágenes de las micrografías de la palma estera confirmaron los buenos resultados en cuanto a las propiedades mecánicas. Por otro lado, fue una de las fibras 


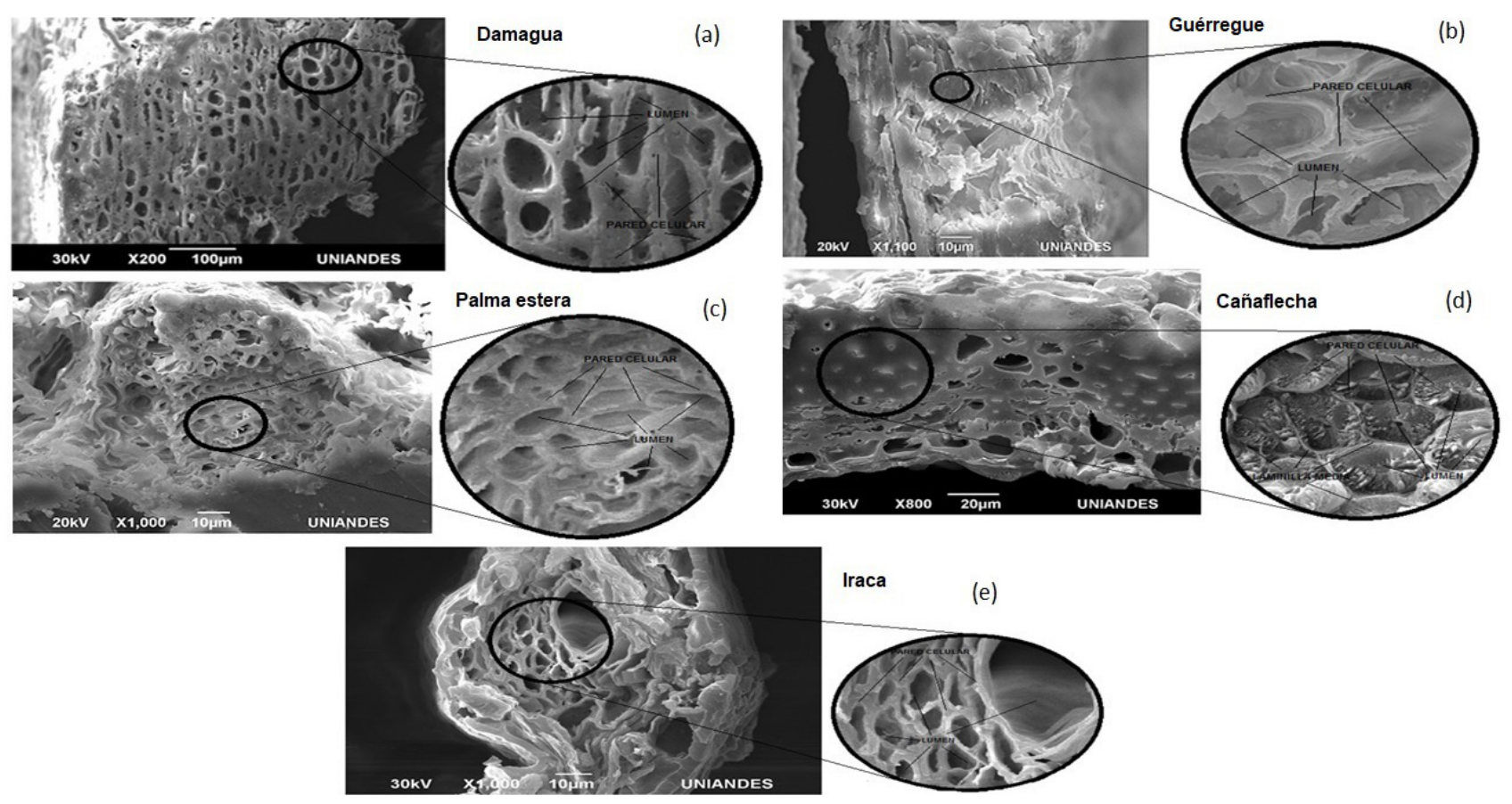

Figura 5. Morfología de la sección transversal de la fibra de: a) la damagua (X200), b) el guérregue (X1100), c) la palma estera (X1000), d) la caña flecha (X800) y e) la iraca (X1000)

con mayor porcentaje de deformación (43\%) debida a la gran pared celular y su laminilla media, lo que permite una delaminación más lenta entre los componentes estructurales, con un comportamiento elástico-plástico (Alves, et al., 2013; Maache, et al., 2017).

La morfología de la fibra de iraca (Figura 5e) mostró variaciones en los diámetros del lumen que oscilaron entre 8 y $30 \mu \mathrm{m}$. La pared celular evidenció tamaños más uniformes, entre 1 y $3 \mu \mathrm{m}$, lo que la convierte en una fibra muy porosa, con un área pequeña para soportar el esfuerzo. La gran diferencia entre en el espesor de la pared celular y el lumen afectó de manera negativa las propiedades mecánicas, y significó que ocupara el cuarto lugar con respecto a la resistencia máxima entre las fibras analizadas.

Las imágenes obtenidas mediante microscopia electrónica de barrido (SEM) evidenciaron características morfológicas similares entre las fibras colombianas analizadas en el presente estudio y otras reportadas en la literatura, como las de yute, sisal, curauá, coco y piassava (Alves, et al., 2013), okra (Abelmoschus esculentus) (DeRosa, et al., 2011), melcocha (Althaea officinalis L) (Sarikanat, et al., 2014), fibras de cáscara de nuez de betel (Areca catechu) (Yusriah, et al., 2014), fibra cabecinegro (Manicaria saccifera) (Porras, et al., 2016), de alcachofa (Cynara cardunculus L.) (Fiore et al., 2011), Juncus effusus L. (Maache, et al., 2017) y fibra de caña de azúcar (Hossain, et al., 2014).

El análisis dela morfología, de las cinco fibras evidenció que la de guérregue y la de caña flecha presentaron paredes celulares más gruesas y lúmenes muy pequeños, lo cual incrementa el área de soporte para una óptima distribución de la carga sobre las microfibrillas de celulosa en la pared secundaria, lo cual les permite un mejor comportamiento mecánico. El caso contrario fue el de las fibras de iraca, damagua y palma estera, cuyos lúmenes presentaron variaciones en el diámetro que afectaron el espesor de la pared celular con la consecuente disminución del área de aplicación y de la resistencia frente a las tensiones axiales en los ensayos de tracción.

\section{Conclusiones}

Se analizaron las propiedades térmicas, mecánicas y morfológicas de las fibras naturales colombianas de damagua, palma estera, caña flecha, iraca y guérregue con el propósito de evaluar su potencial como material de refuerzo en biocompuestos con matrices poliméricas.

El análisis térmico mediante termogravimetría evidenció el rango de temperaturas adecuado para el procesamiento del material compuesto y el uso de matrices poliméricas. El análisis también confirmó el carácter hidrofílico de las fibras naturales, por lo que se sugiere realizar tratamientos superficiales para mejorar la interface con la matriz polimérica.

Son muchos los factores que afectan las propiedades mecánicas de las fibras naturales, entre ellos, la especie de la fibra, el nivel de madurez de la planta y el contenido de celulosa, siendo estos los que definieron las variaciones en las propiedades mecánicas de las fibras evaluadas. Los resultados indicaron que dos de las fibras tienen potencial de alto rendimiento, las de guérregue y caña flecha, lo que las convierte en posibles candidatas para su uso como refuerzo en materiales compuestos. 
La morfología de las fibras visualizada mediante SEM corroboró los valores obtenidos en las pruebas de tracción, en particular la relación entre las paredes celulares y el diámetro del lumen. Las fibras naturales con una pared celular de gran espesor y lúmenes pequeños registraron valores superiores en el comportamiento de sus propiedades mecánicas.

En general, la investigación demostró la importancia de la caracterización de las fibras naturales colombianas, con el fin de explorar alternativas de refuerzo para materiales compuestos con matrices poliméricas y potenciar su uso en aplicaciones industriales.

\section{Agradecimientos}

Al laboratorio de calorimetría y al laboratorio de materiales de la Universidad de Pamplona por toda la colaboración prestada en cada una de las etapas de la investigación.

\section{Información suplementaria}

Tabla 1S. Descripción de las fibras objeto de estudio. Vea la tabla 1S en: https://www.raccefyn.co/index.php/raccefyn/article/ downloadSuppFile/525/2411

Figura 1S. Proceso de extracción de la fibra de la palma guérregue. a) espinas, b) tallo, c) cogollo, d) medialuna de corte, e), f), g) separación de hojas, h) traslado, i) obtención de tiras, $\mathrm{j}, \mathrm{k}$ ) cocido y tinturado, 1) secado, m) entorchado, n) y o) tejido. (Artesanías de Colombia, 2011; Ventas artesanías en guérregue, 2012). Vea la figura 1S en: https://www.raccefyn.co/index.php/raccefyn/article/ downloadSuppFile/525/2403

\section{Conflicto de intereses}

Los autores declaramos que no existe conflicto de intereses de ninguna índole que afecte la publicación de los resultados de este trabajo de investigación.

\section{Contribución de los autores}

WJME adquirió las fibras naturales colombianas y realizó los ensayos y análisis de sus propiedades mecánicas aplicando conceptos de mecánica de materiales. BAR, tutor e investigador, realizó el análisis termogravimétrico y la microscopia electrónica de barrido de las diversas fibras naturales objeto de estudio. Ambos autores redactaron y aprobaron el manuscrito enviado.

\section{Referencias}

Alves, M.E., Castro, T.V., Martins, O.D.F., Silva, F., Toledo, R.D. (2013). The effect of fiber morphology on the tensile strength of natural fibers. J Materials Research and Technology. 2: 149-157.

Arpitha, G.R., Yogesha, B. (2017). An overview on mechanical property evaluation of natural fiber reinforced polymers. Materials today: Proceedings. 4: 2755-2760.

Artesanías de Colombia. Programa de atención a la población desplazada APD. Fecha de consulta: 14 de noviembre de
2014. Disponible en: http://www.artesaniasdecolombia. com.co/PortalAC/C_noticias/artesanias-de-colombiarealiza-capacitacion-a-indigenas-wounaan_1212.

Artesanías de Colombia, ICONTEC, FOMIPYME. (2008). Diagnóstico de la calidad Oficio Artesanal tejeduría. Proyecto de mejoramiento en la calidad y certificacion de productos de artesanos en 13 comunidades ubicadas en diversos departamentos. Aguadas, Caldas. Artesanias de Colombia $.40 \mathrm{p}$

Azwa, Z.N., Yousif, B.F., Manalo, A.C., Karunasena, W. (2013). A review on thedegradability of polymeric composites based on natural fibres. Materials and Design. 47: 424-442.

Balaji, A.N., Nagarajan, K.J. (2017). Characterization of alkali treated and untreated new cellulosic fiber from Saharan aloe vera cactus leaves. Carbohydr Polym. 2017, 174:200208. doi: 10.1016/j.carbpol.2017.06.065.

Bernal, G., Galeano, R. (2013). Cosechar sin destruir. Aprovechamiento sostenible de las palmas colombianas. Bogotá, D.C.: Universiad Nacional de Colombia- Colciencias. ISBN: 978-958-761-611-8. 244p.

Borgtoft Pedersen, H. (1994). Mocora palm fibers: Use and management of Astrocaryum standleyanum (Arecaceae) in Ecuador. Economic Botany. 3: 310-325.

Calderón, E., Galeano, G., García, N. (2005). Libro rojo de las plantas de Colombia. Bogotá, D.C.: Instituto Alexander Von Humbolt-Instituto de Ciencias Naturales, Universidad Nacional de Colombia. Vol 2. 441 p.

Cardona, V., Fuentes, A.F., Cayola, L. (2005). Las moraceas de la región Madidi, Bolivia. Ecología en Bolivia. 40: 212-264.

Castillo, A. (2010). Manual dentrológico de las principales especies de interés comercial actual y potencial de la zona del Alto Huallaga. Lima Perú, Camara Nacional Forestal. 83 p.

Chandramohan, D., Marimuthu K.A. (2011). A Review on natural fibers. IJRRAS. 8: 194-206.

Contreras, M.F., Hormaza, W.A., Marañon, A. (2009). Fractografía de la fibra natural extraída del fique y de un material compuesto reforzado con tejido de fibra de fique y matriz resina poliéster. Suplemento de la Revista Latinoamericana de Metalurgia y Materiales. S1 (1): 57-67.

Cuéllar, A., Muñoz, I. (2010). Fibra de guadua como refuerzo en matrices poliméricas. DYNA. 77: 137-142.

D'Almeida, J.R.M., Aquino, R.C.M.P., Monteiro, SN. (2006). Tensile mechanical properties, morphological aspects and chemical characterization of piassava (Attalea funifera) fibres. Composites Part A: Applied Science and Manufacturing. 37: 1473-1479.

Dai, D., Fan, M. (2010). Characteristic and performance of elementary hemp fibre. Materials Sciences and Applications. 1: 336-342.

Das, S.,Saha AK.,Choudhury PK.,Basak RK.,Mitra BC.,Todd T., Lang, S., Rowell, RM. (2000). Effect of steam pretreatment of jute fiber on dimensional stability of jute composite. J Appl Polymer Science. 76: 1652-1661.

DeRosa, I., Kenny, JM., Maniruzzaman, M., Moniruzzaman, Md., Monti, M., Puglia, D. Santulli, C., Sarasini, F. (2011). Effect of chemical treatments on the mechanical and thermal behaviour of okra (Abelmoschus esculentus) fibres. Composites Science and Technology. 71: 246-254.

Dransfield, J. H. (2015). Royal Botanic Gardens and Ho'omaluhia Botanical Gardens. Fecha de consulta: 13 de noviembre de 2014. Disponible en: http://www.palmpedia.net/wiki/ Astrocaryum_standleyanum 
Elkhaoulani, A., Arrakhiz, F.Z., Benmoussa, K., Bouhfid, R., Qaiss, A. (2013). Mechanical and thermal properties of polymer composite based on natural fibers: Moroccan hemp fibers/polypropylene. Materials and Design. 49: 203-208.

Escobar, E . (2001). Presentación de Yotoco, "Reserva Natural", Flora: plantas vasculares. Palmira. Valle: Universidad Nacional de Colombia, Sede Palmira. 277 p.

Fiore, V., Valenza, A., Di Bella, G. (2011). Artichoke (Cynara cardunculus L.) fibres as potential reinforcement of composite structures. Composites Science and Technology. 71: 1138-1144.

Fiore, V., Scalici T., Valenza, A. (2014). Characterization of a new natural fiber from Arundo donax L. as potential reinforcement of polymer composites. Carbohydrate Polymers. 106: 77-83.

Fuentes, C.A., Willekens, P., Petit, J., Thouminot, C., Müssig J., Trindade L.M., et al. (2017). Effect of he middle lamella biochemical composition on the non-linear behaviour of technical fibres of hemp under tensile loading using strain mapping. Composites: Part A. 101: 529-542.

Galeano, G., Bernal, R. (2010). Palmas de Colombia, Guía de Campo. Bogotá, D.C. Universidad Nacional de Colombia. Ministerio de Ambiente y Desarrollo sostenible. 688 p.

García-Pérez, M., Chaala, A., Yang, J., Roy, C. (2001). Copyrolysis of sugarcane bagasse with petroleum residue. Part I: Thermogravimetric analysis. Fuel. 80: 1245-1258.

Girisha, C.S., Srinivas, GR. (2012). Sisal/coconut coir natural fibers-epoxy composites: Water absorption and mechanical properties. International J Engineering Innovative Techn. 2: 166-170.

Gobernación de Córdoba. (2012). Mejoramiento de la cadena productiva de la cañaflecha (Gynerium sagittatum). Montería, Córdoba: proyecto de investigacion tecnológica. Gobernación de Córdoba. 35 p.

Guo, M., Zhang, T.H., Chen, B.W., Cheng, L. (2014). Tensile strength analysis of palm leaf sheath fiber with Weibull. Composites: Part A. 62: 45-51.

Hossain, MK., Karim, MR., Chowdhury, MR., Imam, MA., Hosur, M., Jeelani, S., et al. (2014). Comparative mechanical and thermal study of chemically treated and untreated single sugarcane fiber bundle. Industrial Crops and Products. 58: 78-90.

Hughes, M. (2012). Defects in natural fibres: Their origin, characteristics and implications for natural fibre-reinforced composites. Journal of Materials Science. 47: 599-609.

Indran, S., Raj, R.E. (2015). Characterization of new natural cellulosic fiber from Cissus quadrangularis stem. Carbohydrate Polymers. 117: 392-399.

Indran, S., Raj, R.E., Sreenivasan, V.S. (2014). Characterization of new natural cellulosic fiber from Cissus quadrangularis root. Carbohydrate Polymers. 110: 423-429.

Ishak, M.R., Sapuan, S.M., Leman, Z., Rahman, M.Z.A., Anwar, U.M.K., Siregar, J.P. (2013). Sugar palm (Arenga pinnata): Its fibres, polymers and composites. Carbohydrate Polymers. 91: 699-710.

Kommula, V.P., Kanchireddy, O.R., Shukla, M., Marwala, T. (2014). Effect of Acid Treatment on the Chemical, Structural, Thermal and Tensile Properties of Napier Grass Fibre Strands. International Conference on Advances in Marine, Industrial and Mechanical Engineering (ICAMIME'2014). Johannesburg (South Africa): 45-51.
Kovacevic, Z., Bischof, S., Fan, M. (2015). The influence of Spartium junceum L. fibres modified with montmorrilonite nanoclay on the thermal properties of PLA biocomposites. Composites Part B. 78: 122-130.

Linares, E., Galeano, G., García, N., Figueroa, Y. (2008.). Fibras vegetales empleadas en Artesanías en Colombia. Bogotá: Artesanías de Colombia, Instituto de Ciencias Naturales, Universidad Nacional de Colombia. 328 p.

Liu, W., Mohanty, A., Drzal, L.T., Misra, M. (2004). Effects of alkali treatment on the structure, morphology and thermal properties of native grass fibers as reinforcements for polymer matrix composites. J Mater Sci. 39: 1051-1054.

Lozano, W.A. (2011). Determinación del número mínimo de observaciones en investigación, obviando las estimaciones de la varianza de datos. Investia y Didactica Ambiental. 10: 54-61.

Maache, M., Bezazi, A., Amroune, S., Scarpa, F., Dufresned, A. (2017). Characterization of a novel natural cellulosic fiber from Juncus effusus L. Carbohydrate Polymers. 171: 163-172.

Maranon, A., Páez, D.C. (2011). Caracterización micromecánica interfacial de un compuesto de ácido láctico reforzado con fibras naturales de cumare. Bogotá: Tesis de Maestria, Facultad de Ingeniería Mecánica. Universidad de los Andes.

Miranda, M.I.G., Bica, C.D.I., Nachtigall, S.M.B., Rehman, N., Rosa, S.M.L. (2013). Kinetical thermal degradation study of maize straw and soybean hull celluloses by simultaneous DSC-TGA and MDSC techniques. Thermochimica Acta. 565: $65-71$.

Mohanty, A.K., Misra, M., Hinrichsen, G. (2000). Biofibers, biodegradable polymers and biocomposites: an overview. Macromol Mat Eng. 276/277: 1-24

Monteiro, SN., Calado, V., Rodríguez, RJS., Margem, FM. (2012). Thermogravimetric behavior of natural fibers rein forced polymer composites-An overview. Materials Science \& Engineering A. 557: 17-28.

Moriana, R., Vilaplanaa, F., Karlssona, S., Ribes, A. (2014). Correlation of chemical, structural and thermal properties of natural fibres for their sustainable exploitation. Carbo hydrate Polymers. 112: 422-431.

Müssig, J. (2010). Industrial Applications of Natural Fibres Structure, Properties and Technical Applications. University of Applied Sciences, Bremen, Germany: John Wiley \& Sons, Ltd. 538 p.

Pickering, K.L., Aruan, M.G., Le, T.M. (2016). A review of recent developments in natural fibre composites and their mechanical performance. Composites: Part A. 83: 98-112.

Pohl, RW. (1983). Gynerium sagittatum (cañaflecha cane). In: D.H. Janzen. Costa Rican natural history. University of Chicago Press. 1: 248-249.

Porras, A., Maranon, A., Ashcroft, I.A. (2016). Characterization of a novel natural cellulose fabric from Manicaria saccifera palm as possible reinforcement of composite materials. Composites Part B: Engineering. 74: 66-73.

Quiñones, C. (2000). El tejido de las tribus indígenas de Colombia. Instituto Colombiano de Cultura Hispánica, Biblioteca Luis Angel Arango. Bogotá. Tomo I: 109-116

Ramírez, G., Klinger, W., Guerra JM., Vargas, L., Valoyes, Z., Robledo, D., Murillo, W., Moreno, N., Cuesta, J.,Cuesta, JJ.,Quezada, Z., Carabali, F. (2011). Plan de manejo del damagua para tres comunidades del Baudó. Quibdó, Chocó. IIAP, Dama-guarte. 89p. 
Rodríguez, L.J., Orrego, C.E., Sarache, W.A. (2014). Elaboración de un material biocompuesto a partir de la fibra de plátano. Manizales: Tesis de Maestria, Universidad Nacional de Colombia. Departamento de Ingeniería Industrial, Manizales, Colombia.

Romero, MH., Maldonado, J., Bogotá, JD., Usma, S., Umaña, AM., Murillo, J., Restrepo, S., Álvarez, M., Palacios, MT, Valbuena, MS.,Mejía, SL, Aldana J. (2009). Informe sobre el estado de la biodiversidad en Colombia 2007-2008: Piedemonte orinoquense, sabanas y bosques asociados al norte del río Guaviare Informe sobre el estado de la biodiversidad en Colombia 2007-2008. Bogotá. Instituto de Investigación de Recursos Biológicos Alexander von Humboldt. $154 \mathrm{p}$

Rong, M.Z., Zhang, M.Q., Liu, Y., Yang, G.C., Zeng, H.M. (2001). The effect of fiber treatment on the mechanical properties of unidirectional sisal-reinforced epoxy composites. Composites Science and Technology. 6: 1437-1447.

Saravanakumar, S.S., Kumaravel, A., Nagarajan, T., Sudhakar, P., Baskaran, R. (2013). Characterization of a novel natural cellulosic fiber from Prosopis juliflora bark. Carbohydrate Polymers. 92: 1928-1933.

Sarikanat, M., Seki, Y., Sever, K., Durmuskahya, C. (2014). Determination of properties of Althaea officinalis L. (Marshmallow) fibres as a potential plant fibre in polymeric composite materials. Composites: Part B. 57: 180-186.
Spinacé, M.A.S., Lambert, C.S., Fermoselli, K.K.G., DePaoli, M.A. (2009). Characterization of lignocellulosic curaua fibres. Carbohydrate Polymers. 77: 47-53.

Srinivasan, V.S., Rajendra S., Sangeetha, D., Vijaya, R. (2014). Evaluation of mechanical and thermal properties of banana-flax based natural fibre composite. Materials and Design. 60: 620-627.

Thygesen, A. (2006). Properties of hemp fibre polymer compositesAn optimisation of fibre properties using novel defibration methods and fibre characterisation. Denmark: Royal Veterinary and Agricultural University. N.11. 148 p.

Ventas artesanías en guérregue. Ventas artesanías en guerregue. Comunidad Woonan. Video promocional. Fecha de consulta: 16 septiembre 2014. Disponible en: www.youtube. com.EYoZ6OfNHgI..

Yang, H., Yan, R., Chen, H., Lee, DH., Zheng, C. (2007). Characteristics of hemicellulose, cellulose and lignin pyrolysis. Fuel. 86: 1781-1788.

Yusriah, L., Sapuan, S,M., Zainudin, E,S., Mariatti, M. (2014). Characterization of physical, mechanical, thermal and morphological properties of agro-waste betel nut (Areca catechu) husk fibre. Journal of Cleaner Production. 72: 174-180 